\title{
Gamma-ray bursts with afterglow plateau phases associated with supernovae
}

\author{
M.G. Dainotti ${ }^{1,2}$, A. D. Boria ${ }^{3}$, J. F. Arratia ${ }^{4}$ \\ ${ }^{1}$ Astronomy Department, Stanford University \\ Stanford, California 94301, USA \\ E-mail: mdainott@stanford.edu \\ ${ }^{2}$ Astronomy Department, Jagellonian Observatory \\ Orla 171, Krakow, 30-244, Poland \\ E-mail: dainotti@uj.edu.pl \\ ${ }^{3}$ Department of Sciences, University of The Sacred Heart, \\ San Juan, Puerto Rico 23173, \\ E-mail: andrea.astrophysics@gmail.com \\ ${ }^{4}$ Department of Sciences, Universidad Metropolitana, \\ San Juan, Puerto Rico 23173, \\ juan.arratia@gmail.com
}

\begin{abstract}
The analysis of 176 GRB afterglow plateaus observed by Swift with known redshifts revealed that the subsample of long GRBs associated with $\mathrm{SNe}$ (GRB-SNe), composed of 19 GRBs, presents a very high correlation coefficient between luminosity at the end of the plateau phase $L_{X}\left(T_{a}\right)=L_{a}$ and the end time of the plateau $T_{a}^{*}$, (hereafter LT correlation). Moreover, a category of GRBs with spectroscopically associated SNe (7 GRBs) show a higher LT correlation than any other analyzed sample, but with a steeper slope than the long GRBs for which no associated SN has been observed (hereafter GRBNO-SNe, 128 GRBs). The difference among the GRB-NO-SNe slope of $128 \mathrm{GRBs}$, and the one of the GRB-SNe (7 GRBs), which we have demonstrated through the Efron \& Petrosian method ${ }^{18}$ not to be due to GRB instrumental selection bias, is statistical significant with $P=0.005$. This possibly suggest that the GRB-SNe might not require a standard energy reservoir in the plateau phase unlike the GRB-NO-SNe. Furthermore, these $\mathrm{SNe} \mathrm{Ib} / \mathrm{c}$ associated with GRBs obey also the peak-magnitude stretch relation, similar to the one used to standardize the SNe Ia. Therefore, this analysis may open new perspective in future theoretical investigations of the GRBs with plateau emission and associated with SNe.
\end{abstract}

Keywords: Gamma-ray bursts.

\section{Introduction}

Notwithstanding the variety of GRB peculiarities, some common features may be identified by looking at their light curves. A crucial breakthrough in this area has been the discovery of the $L_{a}-T_{a}^{*}$ anti-correlation (hereafter LT), Dainotti et al. ${ }^{9}$ proposed to standardize GRBs using afterglow properties, such as the isotropic X-ray luminosity, $L_{a}$ at the time $T_{a}$, the time at the end of the plateau phase $(*$ denotes the rest frame quantities). Later, Dainotti et al. ${ }^{13}$ demonstrated that the LT correlation has an intrinsic slope $b=-1.07_{-0.14}^{+0.09}$ and this finding has an important implication on its possible physical explanation which may imply a fixed energy reservoir powering the plateau. This possibility has been explored in the context of 
the fall-back mass surrounding the Black hole according to the scenario proposed by Cannizzo \& Geherls ${ }^{6}$ and Cannizzo et al. ${ }^{7}$ Also additional theoretical interpretations have been explored to explain the LT correlation, for example the -1 slope, can be derived from a spinning of a newly born magnetar, $2,3,8,23,25,26,28,29$ or due to the prior emission model. ${ }^{30}$ However, there are several models, where generation of this correlation has not been tested yet, such as the photosperic emission model. ${ }^{20}$

In order to test appropriately theoretical models it is also necessary to correctly distinguish among physically different subsamples taken into consideration into the analysis, because if these mentioned subsamples (short with extended emission, for example) are caused by different emission mechanisms this may have consequences on the tightness of the correlation itself and therefore on its use as redshift estimator $^{11}$ as cosmological tool ${ }^{4,5,14,27}$ and as indicator of the ratio between GRB rate and star formation rate. ${ }^{15}$ The problem of selecting homogeneous samples in terms of similar observational properties usually helps to reduce the scatter of correlations, ${ }^{10}$ and it is a general issue which can equally applied to the prompt-afterglow correlations ${ }^{12,16}$ and prompt correlations. ${ }^{1,21,24}$ With this issue in mind we focused on the updated sample of 176 GRBs with known redshift and observed plateau emission looking for a subset with high degree of correlation in the LT space. It is the first time that such an investigation for the LT correlation has been performed considering the GRBs associated with SNe. In the present analysis we use the nomenclature of LONG-SNe and LONG-NO-SNe just for simplicity, since there could be SNe associated with most GRBs, which we did not observe, because no sensitive search was possible at that time or no useful upper limits to the presence of a possible SN in the optical afterglow light curve has been derived. However, we have a couple of counter examples already (while one can indeed be a short GRB) in which we are able to put very stringent limit on the lacks of a supernova emission, such for example the case of long LONG-NO-SNe, 060505 and the case of the short GRB with extended emission, 060614, and possibly a few more GRBs, which are certainly not associated with SNe. From these observations, it seems that the scenario in which long-duration soft-spectrum GRBs are accompanied by massive stellar explosions (LONG-SNe) requires additional explanation for the above events. In summary, this observational panorama is suggestive of the fact that there may be two types of LONG-GRBs with and without SNe. Therefore, it is worthwhile to investigate a reasonable distinction in these categories to better clarify such a debated issue. Within this context our categorization of LONG-SNe becomes an observational homogeneous motivated sample. Since the LT relationship is connected to the physics of the GRB and it has been used as model discriminator, it is worth asking whether there is evidence that the relationship is notably different for GRBs with and without SNe.

Below, in Sec. 2 we describe data analysis, in Sec. 3 we divide the total sample into categories, such as in long GRBs with evidence of association with SNe (LONG$\mathrm{SNe}$ ), long GRBs with no evidence of associated SNe (LONG-NO-SNe), short GRBs 
with extended emission, (SE) and X-Ray Flashes (XRFs). In Sec. 4 we present summary and main conclusions.

\section{Data analysis}

We analyzed the sample of all (176) GRB X-ray afterglows, detected by Swift from January 2005 up to July 2014 with known redshifts for which the light curves can be fitted by the Willingale et al. ${ }^{31}$ hereafter W07, phenomenological model. We use the redshifts available in the literature, looking at the Greiner web page ${ }^{\mathrm{a}}$ and in the Circulars Notice arxive (GCN). The redshift range of our sample is $(0.033,9.4)$.

We compute the source rest-frame luminosity $L_{a}$ in the Swift XRT band pass, $\left(E_{\text {min }}, E_{\text {max }}\right)=(0.3,10) \mathrm{keV}$ as following Dainotti et al. ${ }^{13}$ The normalization, $a$, and slope, $b$, of the LT correlation for the distributions of the all analyzed subsamples, see Sec. 3, have been derived using the D'Agostini method fitting procedure. ${ }^{17}$
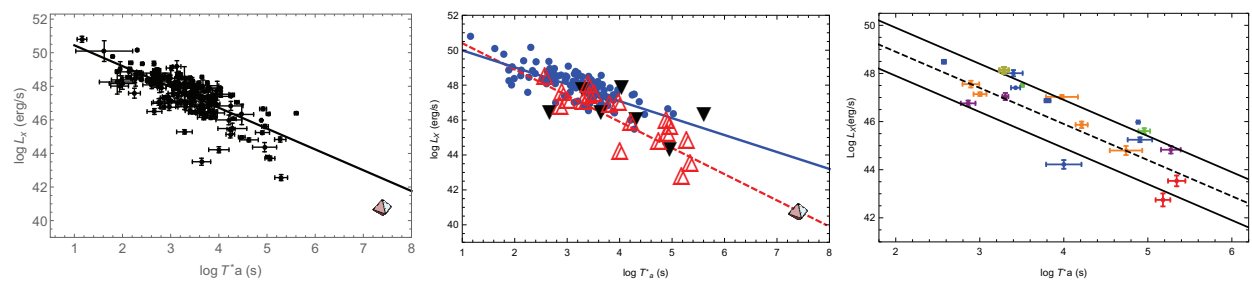

Fig. 1. The analyzed $\log L_{X}$ vs. $\log T_{a}^{*}$ distributions. Left panel: the total sample of 176 GRB afterglows with the correlation fit given by a solid line, the diamond symbol represents GRB 980425. Middle panel: LONG-NO-SNe 128 GRBs (blue points fitted with a solid blue line), LONG-NO-SNe at small redshift, $z \leq 0.79$, (black filled triangles) and the 19 events from LONGSNe (red empty triangles) fitted with a red dashed line. Right panel: The analyzed $\log L_{X}$ vs. $\log T_{a}^{*}$ distributions for LONG-SNe divided in colors depending on the category described in Table 2. Category A: red points; B: orange; C: green; D: purple; E: blue. The dark yellow point represent a low redshift GRB, GRB 060505, for which the SN was not seen associated to GRB, see discussion in the text. The two solid lines represent the $1 \sigma$ intrinsic scatter of the LT correlation.

\section{The LT correlation in GRB subsamples}

With the aim of finding common trends among the selected 176 GRB lightcurves, all observed by Swift (see left panel of Fig. 1), we analyze the subsamples of 160 GRB-LONG, which is the total sample subtracted of the 16 short GRBs with extended emissions (LONG-SE). Within the LONG sample we analyze 19 GRBsSNe, red empty triangles in right panel of Fig. 1, 128 LONG-NO-SNe, blue points in right panel of Fig. 1 and $25 \mathrm{X}$-ray Flashes (XRFs). 12 GRBs are common in the LONG-SNe and XRFs samples. Within the LONG-SNe sample we applied a further

\footnotetext{
ahttp://www.mpe.mpg.de/ jcg/grbgen.html
} 
classification, which is an update of the classification of Hjorth \& Bloom. ${ }^{19}$ This classification identifies a 'standard' sample of LONG-SNe with common properties, namely subsamples of the LONG-SNe based on the quality of the identification of SN associated to the GRB. The considered categories are: A) strong spectroscopic evidence for a SN associated with the GRB, B) a clear light curve bump as well as some spectroscopic evidence suggesting the LONG-SN association, C): a clear bump on the lightcurve consistent with the LONG-SN association, but no spectroscopic evidence of the SN, D) a significant bump on the lightcurve, but the inferred SN properties are not fully consistent with other LONG-SN associations or the bump is not well sampled or there is no spectroscopic redshift of the GRB, E) a bump, either of low significance or inconsistent with other observed LONG-SN identifications, but with a spectroscopic redshift of the GRB.

For all the subsamples the Spearman correlation coefficient, $\rho_{L T}$, is always greater than 0.5 and the probability that the fitted correlation occurred randomly in an uncorrelated data set is $P<0.05$, thus confirming the existence of these correlations.

\subsection{GRBs associated with SNe}

Therefore, within the LONG-SNe sample we propose a further division, which is an update of the classification of Hjorth \& Bloom. ${ }^{19}$ This classification identifies a 'standard' sample of LONG-SNe with common properties, namely subsamples of the LONG-SNe based on the quality of the identification of SN associated to the GRB. The considered categories are: A) strong spectroscopic evidence for a SN associated with the GRB, B) a clear light curve bump as well as some spectroscopic evidence suggesting the LONG-SN association, C): a clear bump on the lightcurve consistent with the LONG-SN association, but no spectroscopic evidence of the SN, D) a significant bump on the lightcurve, but the inferred SN properties are not fully consistent with other LONG-SN associations or the bump is not well sampled or there is no spectroscopic redshift of the GRB, E) a bump, either of low significance or inconsistent with other observed LONG-SN identifications, but with a spectroscopic redshift of the GRB.

We present the fitted correlation slope, $b$ and its error $\delta_{b}$, the normalization, $a$, and its error, $\delta_{a}$, the $\rho_{L T}$, and the probability $P$ for all the analyzed subsample, see Table 1, Fig. 1.

To further discriminate GRBs within the LONG-SNe sample, we divided it into 5 subcategories that distinguish spectroscopic characteristics of the SN associated with the GRB.

Our Swift LONG-SNe sample has $\rho_{L O N G-S N e}=-0.83$, higher than the LONGNO-SNe sample computed in the same redshift range of the LONG-SNe with $\rho_{L O N G-N O-S N e}=-0.66$. The $\mathrm{A}$ and $\mathrm{B}$ categories together (7 GRBs) present $\rho_{L O N G-S N e_{A+B}}=-0.96$, higher than the $\rho_{L O N G-N O-S N e}=-0.66$, with a probability $P=3.0 \times 10^{-4}$, thus confirming the existence of this tight correla- 
Table 1. The analyzed GRB samples. In the successive columns the table shows a GRB sample, a number $\mathrm{N}$ of events in the sample, the fitted correlation slope, $b$ and its error $\delta_{b}$, the intercept, $a$, and its error, $\delta_{a}$, the Spearman correlation coefficient, $\rho_{L T}$, and the probability $P$.

\begin{tabular}{|l|l|l|l|l|l|}
\hline GRB sample & $\mathrm{N}$ & $b \pm \delta_{b}$ & $a \pm \delta_{a}$ & $\rho_{L T}$ & $P$ \\
\hline All & 176 & $-1.2 \pm 0.1$ & $51.6 \pm 0.3$ & -0.74 & $4 \cdot 10^{-32}$ \\
Long-NO-SNe & 128 & $-1.0 \pm 0.1$ & $51.0 \pm 0.3$ & -0.74 & $9 \cdot 10^{-24}$ \\
Long-NO-SNe $(z \leq 1.16)$ & 19 & $-0.8 \pm 0.2$ & $49.9 \pm 0.9$ & -0.66 & $1 \cdot 10^{-3}$ \\
Long-NO-SNe $(z \leq 1.06)$ & 18 & $-0.8 \pm 0.2$ & $49.8 \pm 1.0$ & -0.66 & $1 \cdot 10^{-3}$ \\
LONG-SNe & 19 & $-1.5 \pm 0.3$ & $51.9 \pm 1.0$ & -0.83 & $5 \cdot 10^{-6}$ \\
LONG-SNe $(z \leq 0.937)$ & 18 & $-1.5 \pm 0.3$ & $51.7 \pm 1.0$ & -0.84 & $6 \cdot 10^{-6}$ \\
LONG-SNe (A+B) & 7 & $-1.9 \pm 0.3$ & $53.1 \pm 1.3$ & -0.96 & $3 \cdot 10^{-4}$ \\
LONG-NO-SNe $(z \leq 0.79)$ & 7 & $-0.5 \pm 0.5$ & $48.7 \pm 2.1$ & -0.57 & 0.09 \\
SE & 16 & $-1.4 \pm 0.3$ & $51.3 \pm 1.0$ & -0.71 & $1 \cdot 10^{-6}$ \\
XRF & 25 & $-1.6 \pm 0.3$ & $52.8 \pm 1.0$ & -0.72 & $1 \cdot 10^{-6}$ \\
\hline
\end{tabular}

tion. The values of correlation coefficients presented in Table 1 show a 'maximal' $\rho_{L O N G-S N e(A+B)}=-0.96$ leading to the conclusion that the best correlated sample has a clear identification of the underlying supernovae.

The high $\rho_{L T}$ for the LONG-SNe sample shows how on the basis of only obeying the LT correlation, without any further selection criterion, we are able to select an homogeneous and observational motivated subsample of GRBs. Indeed, before arriving to the conclusion that the LONG-SNe sample has the highest correlation coefficient, we tried several classifications based on the morphological structure of the lightcurves, for example the $\chi^{2}$ of the fitted plateau or the flatness of the plateau itself. We conclude that the LONG-SNe subsample seems a better choice than any other one selected on the basis of the morphology, spectral features of the lightcurves and on the fitting parameters.

\section{Conclusions}

From Table 1 we note that the slope of the $(\mathrm{A}+\mathrm{B})$ differs $2.8 \sigma$ from the slope of the LONG-NO-SNe sample. However, for a more appropriate comparison we considered two samples observed in the exact redshift range and with the same number of events. We confirm that this difference is the same also when we correct the total 128 GRBs for redshift evolution and selection effects, for details see Dainotti et al. ${ }^{13}$ This means that this difference is statistically significant with the same probability resulting from the T-student test, $P=0.005$. Therefore, this indicates from a physical point of view that if the correlation slope not anymore -1.0 this may not guarantee any more that the energy reservoir in the plateau remains constant. This condition instead is valid for the all sample of LONG-NO-SNe. This evidence might possibly lead to a different theoretical interpretation for the plateau phase of the LONG-SNe sample. 


\section{Acknowledgments}

This work made use of data supplied by the UK Swift Science Data Centre at the University of Leicester. M.G.D is grateful to the Marie Curie Program, because the research leading to these results has received funding from the European Union Seventh FrameWork Program (FP7-2007/2013) under grant agreement N 626267.

\section{References}

1. Amati, L., Frontera, F. \& Guidorzi, C., A\&A, 508, 173.

2. Bernardini, M.G. et al. 2011, accepted on A\&A arXiv 1112.1058B

3. Bernardini, M.G. et al. 2012, MNRAS, 425, 1199B

4. Cardone, V.F, Capozziello, S. \& Dainotti, M.G., 2009, MNRAS, 400, 775

5. Cardone, V.F., Dainotti, M.G., et al. 2010, MNRAS tmp 1386C

6. Cannizzo, J. K. \& Gehrels, N., 2009, ApJ, 700, 1047

7. Cannizzo, J. K., Troja, E. \& Gehrels, N., 2011, ApJ, 734, 35C

8. Dall'Osso, S. et al. 2011, A\&A, 526A, 121D

9. Dainotti, M. G., Cardone, V. F. and Capozziello, S. 2008, MNRAS 391L, 79D

10. Dainotti, M.G., et al. 2010, ApJL, 722, L215

11. Dainotti, M. G., et al., 2011, ApJ, 730, 135D

12. Dainotti, M.G., M. Ostrowski \& Willingale, R., 2010, MNRAS, 418, 2202D

13. Dainotti, M.G., et al. 2013, ApJ, 774, 157

14. Dainotti, M.G., et al. 2013, MNRAS, 436, 82

15. Dainotti, M.G., et al. 2015, ApJ, 800, 31D

16. Dainotti, M.G., et al. 2015, MNRAS, 451, 4

17. D'Agostini, G. 2005, arXiv: physics/0511182

18. Efron, B. \& Petrosian, V., 1992, ApJ, 399, 345

19. Hjorth, J., \& Bloom, J.S, 2011, 'Gamma-Ray Bursts", eds. C. Kouveliotou, R. A. M. J. Wijers, S. E. Woosley, Cambridge University Press, 2011.

20. Ito et al. 2014, ApJ, 789, 159

21. Lloyd, N., \& Petrosian, V. ApJ, 2000, 543, 722L

22. O'Brien, P.T., Willingale, R., Osborne, J. et al. 2006, ApJ, 647, 1213

23. O'Brien, P.T. \& Rowlinson, A., 2012, IAUS, 279, $297 \mathrm{O}$

24. Qi, S \& Lu, T. 2012, ApJ, 749, 99Q

25. Rowlinson, A. et al. 2010, AIP Conference Proceedings, Volume 1358

26. Rowlinson, A., et al. 2014, MNRAS, 443, 1779

27. Postnikov, S., Dainotti, M.G., Hernandez, X. \& Capozziello, S., ApJ submitted.

28. Rea, N. et al. 2015 ApJ, 813, 92

29. Nemmen, R. S., et al. 2012, Science, 338, 6113, 1445

30. Yamazaki, R. 2009, Apj, 690, L118

31. Willingale, R.W. et al., ApJ, 2007, 662, 1093 